\title{
Anomalous diffusion in the resonant quantum kicked rotor
}

\author{
A. Romanelli, Guzmán Hernández \\ Instituto de Física, Facultad de Ingeniería \\ Universidad de la República \\ C.C. 30, C.P. 11000, Montevideo, Uruguay \\ e-mail: alejo@fing.edu.uy,guzmanhc@fing.edu.uy
}

\begin{abstract}
We study the resonances of the quantum kicked rotor subjected to an excitation that follows a deterministic time-dependent prescription. For the primary resonances we find an analytical relation between the long-time behavior of the standard deviation and the external kick strength. For the secondary resonances we obtain essentially the same result numerically. Selecting the time sequence of the kick allows to obtain a variety of asymptotic wave-function spreadings: super-ballistic, ballistic, sub-ballistic, diffusive, sub-diffusive and localized.
\end{abstract}

Key words: Quantum resonance; Kicked rotor; Anomalous diffusion

\section{Introduction}

The kicked rotor model is a paradigm of periodically driven systems with classical chaotic behavior and its quantum version, the quantum kicked rotor (QKR), can be considered a cornerstone in the study of chaos at the quantum level 11. This topic is a matter of permanent attention, both theoretical and experimental, for example it has been recently reported [2] the experimental realization of the quantum kicked top (a variant of the QKR.) to study signatures of quantum chaos.

The behavior of the QKR has two characteristic modalities: dynamical localization and ballistic spreading of the variance in resonance [3]. These behaviors are quite different and have no classical analog. They depend on the relationship between the characteristic time of the free rotor and the period associated to the kick. When the dimensionless period of the kick, $T$, is an irrational multiple of $2 \pi$ the average energy of the system grows in a diffusive manner for 
a short time and afterwards dynamical localization appears. In this case, the angular momentum distribution has a maximum at its mean value and decays exponentially. For $T$ rational multiple of $2 \pi$ the behavior of the system is resonant with ballistic spreading, and the angular momentum distribution evolves from the initial one in such away that its standard deviation has the time dependence $\sigma(t) \sim t$. The two types of values of $T$ determine the spectral properties of the Hamiltonian, for irrational multiples the energy spectrum is purely discrete and for rational multiples it contains a continuous part. Both quantum resonance and dynamical localization can be seen as interference phenomena, the first being a constructive interference effect and the second a destructive one.

Different variants of the QKR have been studied, but with the aim of this work in mind, it is interesting to highlight the one developed by [4,5] where the QKR is subjected to two unitary operators with two different values of the strength parameter $K$. The dynamics of the QKR kicked according to a Fibonacci sequence is studied in Ref. [4. This is done outside the resonant regime, where a sub-diffusive behavior is found for small kicking strengths. In Ref. [5] we have also studied the QKR excited with the same Fibonacci prescription but in the resonant regime. It was shown that the standard deviation of the primary resonances maintains the well-known ballistic behavior but the secondary resonances have sub-ballistic behavior, characterized by the time dependence of its standard deviation $\sigma(t) \sim t^{\gamma}$, with $1 / 2<\gamma<1$. This change of behavior is similar to that obtained for the quantum walk (QW) subjected to an aperiodic Fibonacci excitation [6] where the ballistic behavior is lost and the sub-ballistic behavior appears. We have also investigated the QKR in resonance subjected to decoherence: a) with a Lévy waiting-time distribution [7,8] and b) with an unitary noise defined through Kraus operators [9]. In both cases we find that the secondary resonances have a sub-ballistic behavior, while the principal resonances are not affected. On the other hand, in Refs. [10,11] the QKR subjected to noises with a Lévy distribution out of resonance was investigated, showing that the decoherence leads to a sub-diffusive regime $(\gamma<1 / 2)$ for a short time before localization appears.

The quantum resonances and the dynamical localization have been experimentally observed in Refs. [12,13,14,15]. The QKR was experimentally realized through a dilute sample of ultra-cold atoms exposed to a one-dimensional spatially periodic optical potential that is pulsed on periodically in time (to approximate a series of delta function kicks). However, the ballistic growth of the secondary resonances was not confirmed. It was found that the momentum distribution profiles saturated to a final distribution but different from the characteristic exponential profiles of the dynamical localization. In Ref. [13] the usual QKR is modified, by adding other elements such as a quasimomentum, in order to explain the absence of the ballistic growth. However later experiments [13] has confirmed this growth. 
In a recent paper [17] we have studied the QW model using a time-dependent unitary coin operator. We found different types of anomalous diffusion depending on the time prescription for the coin operator. In the same line of thought, in this paper we develop a simple QKR model with a generalized time strength parameter $K(t)$ that allows an analytical treatment of the primary resonances. We study the connection between the time evolution of $K(t)$ and the type of spreading of the system. We analytically show how to select the time dependence of $K(t)$ to obtain a predetermined power-law distribution of the standard deviation. We propose this toy model to reproduce the experimental behavior reported in Refs. [13]15].

The paper is organized as follows: In the next section we present a brief revision of the QKR equations, in the third section we obtain analytically the standard deviation for the primary resonances, in section $I V$ we choose a time dependence for $K(t)$, in section $V$ numerical results are presented for the secondary resonances, and in the last section we draw the conclusions.

\section{Quantum kicked rotor}

The QKR is one of the simplest and best investigated quantum models whose classical counterpart displays chaos. In this paper we use a modified version of the usual QKR where a deterministic time dependence for the strength parameter $K(t)$ is introduced. The Hamiltonian has then the following shape

$$
H(t)=\frac{P^{2}}{2 I}+K(t) \cos \theta \sum_{n=1}^{\infty} \delta(t-n T),
$$

where the external kicks occur at times $t=n T$ with integer $n, T$ being the kick period (the index $n$ will be equivalent to time in units of $T$, given the delta function appearing in Eq.(1) ), $I$ is the moment of inertia of the rotor, $P$ the angular momentum operator and $\theta$ the angular position operator. The last two operators satisfy the canonical commutation rule

$$
[P, \theta]=-i \hbar
$$

In the angular momentum representation, $P|l\rangle=l \hbar|l\rangle$, the wave-vector is

$$
|\Psi(n)\rangle=\sum_{l=-\infty}^{\infty} a_{l}(n)|l\rangle
$$

and the average energy is

$$
E(n)=\langle\Psi|H| \Psi\rangle=\varepsilon \sum_{l=-\infty}^{\infty} l^{2}\left|a_{l}(n)\right|^{2},
$$


where $\varepsilon=\hbar^{2} / 2 I$. Using the Schrödinger equation the quantum map is readily obtained from the Hamiltonian Eq.(11)

$$
a_{l}(n+1)=\sum_{j=-\infty}^{\infty} U_{l j} a_{j}(n)
$$

where the matrix element of the time step evolution operator $U(\kappa)$ is

$$
U_{l j}=i^{-(j-l)} e^{-i j^{2} \varepsilon T / \hbar} J_{j-l}(\kappa(n)),
$$

$J_{l}$ being the $l$ th order cylindrical Bessel function and its argument the dimensionless kick strength $\kappa(n) \equiv K(n) / \hbar$. With the aim to generate the dynamics of the system we will consider different time dependencies of the strength parameter and combine the corresponding time-step operators $U(\kappa(n))$ in a large sequence. The resonance condition does not depend on $\kappa$ and takes place when the frequency of the driving force is commensurable with the frequencies of the free rotor. Inspection of Eq.(6) shows that the resonant values of the scale parameter $\tau \equiv \varepsilon T / 2 \hbar$ are the set of the rational multiples of $2 \pi, \tau=2 \pi p / q$. In what follows we assume, that the resonance condition is satisfied, therefore the evolution operator depends on $\kappa, p$ and $q$. We call a resonance primary when $p / q$ is an integer and secondary when it is not.

\section{Primary resonances}

We shall first consider the primary resonances. Using the recursion relation satisfied by the Bessel functions Eq.(5) is solved. Its general solution is written as

$$
a_{l}(n)=\sum_{j=-\infty}^{\infty}(-i)^{l-j} a_{j}(0) J_{l-j}\left(n^{*}\right),
$$

where $a_{j}(0)$ are the initial amplitudes and

$$
n^{*}=\sum_{m=1}^{n} \kappa(m)
$$

From Eq.(7) it is clear that the propagation speed of the probability amplitudes is given by $\Delta n^{*} / \Delta n=\kappa(n)$. The probability distribution at time $n$ is given by

$$
P_{l}(n)=\left|a_{l}(n)\right|^{2}
$$

that can be expressed as

$$
P_{l}(n)=\sum_{j, k=-\infty}^{\infty}(-i)^{k-j} a_{k}(0) a_{j}^{*}(0) J_{l-k}\left(n^{*}\right) J_{l-j}\left(n^{*}\right) .
$$


Using the properties of the Bessel functions all the moments of $P_{l}(n)$ can be calculated analytically from Eq.(10). If symmetrical initial conditions are taken, the odd moments vanish due to the symmetrical dependence on the initial conditions. We calculate the first and second moments, obtaining:

$$
\begin{aligned}
& M_{1}(n)=-n^{*} \sum_{j=-\infty}^{\infty} \Im\left[a_{j}(0) a_{j-1}^{*}(0)\right]+M_{1}(0), \\
M_{2}(n)= & \frac{\left(n^{*}\right)^{2}}{2}\left(1-\sum_{j=-\infty}^{\infty} \Re\left[a_{j}(0) a_{j+2}^{*}(0)\right]\right) \\
& +n^{*} \sum_{j=-\infty}^{\infty}(2 j+1) \Im\left[a_{j}(0) a_{j+1}^{*}(0)\right] \\
& +M_{2}(0)
\end{aligned}
$$

where $\Re[x]$ and $\Im[x]$ are the real and imaginary part of $x$ respectively. The standard deviation $\sigma=\sqrt{M_{2}-M_{1}^{2}}$ has the following long-time limit

$$
\sigma(n) \rightarrow C n^{*}=C \sum_{m=1}^{n} \kappa(m)
$$

where $C$ is a constant that only depends on the initial conditions.

\section{Choosing $K(t)$}

Among the alternatives to choose the time dependence of $\kappa(n)$ we study the case:

$$
\kappa(n)=\kappa_{0}\left(\frac{1}{n}\right)^{\alpha}
$$

where $\alpha$ is a real parameter and $\kappa_{0}$ is a constant. Substituting Eq.(14) in Eq.(13) and omitting a multiplicative constant the following asymptotic results are obtained

where

$$
\sigma(n) \rightarrow\left\{\begin{array}{l}
\ln n, \text { if } \alpha=1, \\
n^{\gamma}, \text { if } \alpha \neq 1,
\end{array}\right.
$$

$$
\gamma \equiv 1-\alpha
$$

The asymptotic spreading of the wave function shows seven different behaviors that can be characterized by the exponent $\gamma$ : 
(a) $\gamma>1$, super-ballistic.

(b) $\gamma=1$, ballistic.

(c) $1>\gamma>1 / 2$, sub-ballistic.

(d) $\gamma=1 / 2$, diffusive.

(e) $1 / 2>\gamma>0$, sub-diffusive.

(f) $\gamma=0$, logarithmic.

(g) $\gamma<0$, Bessel-localized.

The result for $\gamma=1$ is expected because the system reduces to the usual QKR in resonance. In the other cases the system shows unexpected behaviors. For $\gamma<0$ the system does not spread when $t \rightarrow \infty$, it becomes localized in angular momentum. We have called this behavior 'Bessel-localized' because the distribution decay is slower than the case of exponential localization, as can be seen from Eq.(10). Note that this equation with initial conditions $a_{k}(0)=$ $\delta_{k m}$ is reduced to:

$$
P_{l}(n)=\left[J_{l-m}\left(n^{*}\right)\right]^{2} .
$$

In the other extreme for $\gamma>1$ the spreading of the wave-function is superballistic. In this case not only the external frequency matches the natural frequency of the free rotator (resonance condition) but the intensity of the external strength grows with time as well. We could think that this condition produces a new kind of reinforced resonance. In order to visualize the asymptotic behavior of the distribution $P_{l}$, we use Eq.(17) to calculate the profiles of the distribution. These profiles are shown in Fig. 1 for several values of $\alpha$ after a time long enough has elapsed, to see the spreading of the distribution. We take the initial conditions $a_{l}(0)=\delta_{l 0}$, to produce a symmetrical evolution. From this figure, it is clear that as $\alpha$ grows the spreading speed decreases, the distribution shrinks and the two extreme peaks come closer. When $\alpha=1 / 2$ the standard deviation spreads out as $\sigma(t) \sim t^{1 / 2}$, but the probability distribution $P_{l}$ is not Gaussian. This confirms that the evolution corresponds to a coherent unitary process and not to the classical diffusive behavior. In the extreme case when $\alpha>1$ the distribution is restricted to a small interval around its initial value and the two extreme peaks melt into a very narrow peak. It is important to point out that the distribution profiles presented here are qualitatively similar to those obtained in Ref. [16]17] for the QW.

To conclude this section, it is important to point out that the specific form of the kick sequences in Eq.(14) is not essential to obtain the qualitative behaviors described above, they are independent of this choice. Note that $\sigma(n)$ is determined by Eq.(13) and the qualitative behavior of $\kappa(n)$. If $\kappa(n) \rightarrow 0$, $\sigma(n)$ cannot grow faster than in the ballistic case, therefore it behaves as in 


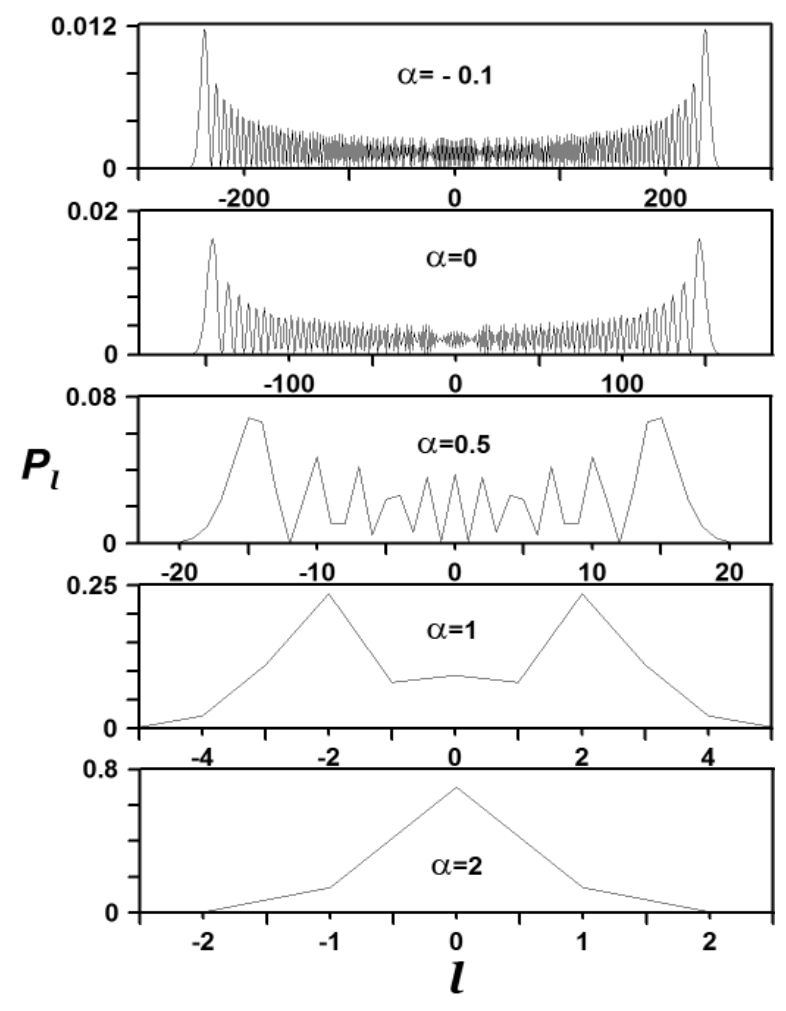

Fig. 1. The probability distribution $P_{l}$, for a primary resonance as a function of the dimensionless momentum $l$ at time $n=300$ with the initial condition $a_{l}(0)=\delta_{l 0}$. The values of the parameter $\alpha$ are, from top to bottom, $-0.1,0,0.5,1$ and 2 .

the cases denoted above by (b), (c), (d), (e) or (g), depending on the rate of decay of $\kappa(n)$. When $\kappa(n) \nrightarrow 0$ but remains bounded, the behavior is ballistic. Finally when $\kappa(n)$ is not bounded, $\sigma(n)$ grows super-ballistically as in case (a).

\section{Secondary resonances}

Now we inquire about the secondary resonances of the QKR. In the general case the analytical development is very cumbersome because the matrix elements of the unitary map in Eq.(6) include the time dependent phases $e^{-i j^{2} \varepsilon T / \hbar}$. These phases impede the integrability of the problem. It could be possible to obtain analytical results for some special function $\kappa(t)$ following the work of Ref. [18]. However, we opt to study the secondary resonances numerically iterating Eq.(5) with the time dependent kick proposed in Eq.(14) to calculate the distribution $P_{l}$ and the standard deviation. In Fig. 2 the standard deviation $\sigma$ for the secondary resonance $p / q=2 / 5$ is presented for several values of the parameter $\alpha$. It is seen that $\sigma$ has a power-law growth with an exponent $\gamma$ that depends on $\alpha$, but now the relation given by Eq.(16) does not hold. The values of $\gamma$ were adjusted for the last hundred values of $n$. The 


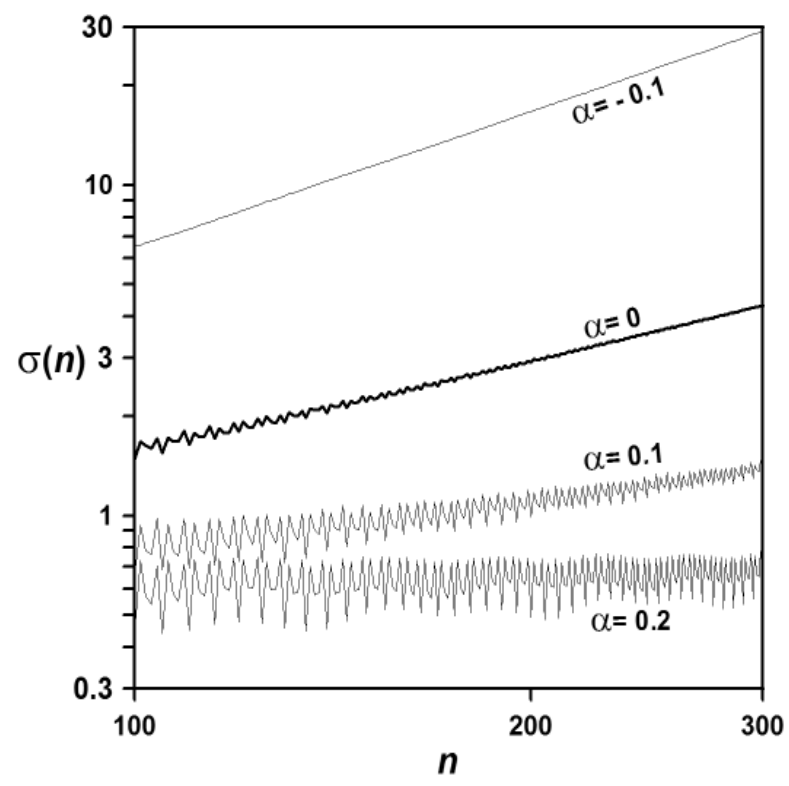

Fig. 2. The standard deviation $\sigma(n)$ as a function of the dimensionless time $n$ in $\log$-log scales for the secondary resonance $p / q=2 / 5$. The initial condition for the amplitudes is $a_{l}(0)=\delta_{l 0}$. For large $n$ the curves satisfy, on the average, a power law $n^{\gamma}$. The values of $\gamma$ are, from top to bottom, (1) $\gamma \sim 1.4$ for $\alpha=-0.1$, (2) $\gamma=1$ for $\alpha=0,(3) \gamma \sim 0.5$ for $\alpha=0.1$, and (4) $\gamma \sim 0$ for $\alpha=0.2$.

ranges of $\alpha$ for the different behaviors are now the following:

(a) $\alpha<0$, super-ballistic $(\gamma>1)$,

(b) $\alpha=0$, ballistic $(\gamma=1)$,

(c) $0<\alpha \lesssim 0.1$, sub-ballistic $(1>\gamma \gtrsim 0.5)$,

(d) $\alpha \simeq 0.1$, diffusive $(\gamma \simeq 0.5)$

(e) $0.1 \lesssim \alpha \lesssim 0.2$, sub-diffusive $0.5 \gtrsim \gamma \gtrsim 0$,

(f) $\alpha \gtrsim 0.2$, localized $(\gamma \lesssim 0)$.

If we compare these ranges with the corresponding ranges of $\alpha$ for the primary resonances we find a similar qualitative behavior, as in the cases (a), (b), (c), (d) and (e). However in the present calculation, the logarithmic case is not present and it is not easy to classify the type of localization obtained.

In Fig. 3 we present the distribution $P_{l}$ as a function of the angular momentum for four values of $\alpha$. The distributions show a symmetrical spreading around a central peak. The spreading shrinks as $\alpha$ grows, going towards the localized behavior, as in the case of the primary resonances. 


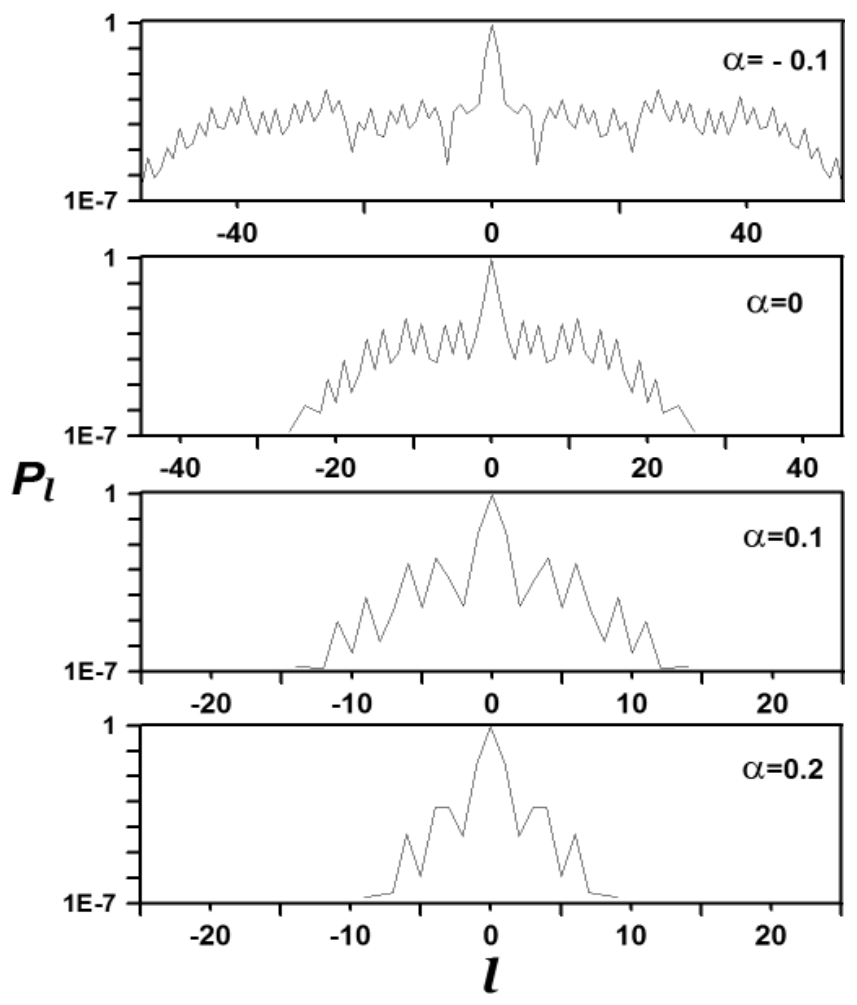

Fig. 3. The probability distribution $P_{l}$, for the secondary resonance $p / q=2 / 5$, as a function of the dimensionless momentum $l$ at time $n=300$ for the initial condition $a_{l}(0)=\delta_{l 0}$. The vertical scale is logarithmic.

\section{Discussion and conclusion}

The resonances of the QKR, as well as the dynamical localization, were first established numerically [1] and the analytical treatment of the resonances was developed by Izrailev and Shepelyansky [18]. Thirteen years ago the primary resonances were experimentally observed by Bharucha et al. [13], in samples of cold atoms interacting with a far-detuned standing wave of laser light. More recently Kanem et al. [15] have observed secondary resonances. These notable series of experiments, together with those in Refs. [12]14]19], have drawn much attention because they have allowed to study fundamental aspects of the interaction between matter and radiation, and may also contribute to the experimental basis of quantum computation. In Ref. [13] the usual QKR model was used to describe the experimental results where dynamical localization was found out of resonance. However, they did not observe the ballistic growth in resonance predicted by the model. Instead, an unexpected decay of the distribution was obtained. The variety of anomalous behaviors of the resonances presented in this paper could be useful to interpret these experimental results.

On the other hand, the experimental set up described in the previous references 
could be used for the realization of the QKR proposed in this work. The most important difference with the realized experiments would reside in that the intensity of the laser that hits the cloud of atoms should depend on time.

In summary, we have developed a variant of the QKR model that shows anomalous diffusion in resonances. The spreading of the wave function has a rich variety of behaviors that go from the localized to the super-diffusive. This feature opens interesting possibilities for quantum information processing because it could be used to control the spreading of the wave function. For the primary resonances, in the long-time asymptotic regime, we have found an analytical expression for the dependence of the standard deviation with the strength parameter $K(t)$. This expression shows explicitly how to select the unitary evolution to obtain a predetermined asymptotic behavior for the wave function spreading: super-ballistic, ballistic, sub-ballistic, diffusive, subdiffusive, logarithmic or Bessel-localized. The parameter $\alpha$ determines the degree of diffusivity of the system in the long-time limit. We have shown numerically that the standard deviation for the secondary resonances has the same qualitative behavior as that exhibited by the primary resonances. The probability distributions are similar in the case of localization for both types of resonances, but in the other cases the profiles are very different.

Finally, it is important to mention that there exists a parallelism between the results obtained in the present work and those obtained in Ref. [17] for the QW. This evidences the theoretical equivalence between the QKR in resonance and the QW [7,21,20].

We acknowledge the support from PEDECIBA, ANII and thank V. Micenmacher for his comments and stimulating discussions.

\section{References}

[1] G. Casati, B.V. Chirikov, F.M. Izrailev, and J. Ford, Lect. Notes Phys. 93, 334 (1979).

[2] S. Chaudhury, A. Smith, B. E. Anderson, S. Ghose, and P. S. Jessen, Nature 461, 768, (2009).

[3] F. M. Izrailev, Phys. Rep. 196, 299 (1990).

[4] G. Casati, G. Mantica and D.L. Shepelyansky Phys. Rev. E 63, 066217 (2001).

[5] A. Romanelli, A. Auyuanet, R. Siri and V. Micenmacher. Phys. Lett. A, 365, 200 (2007).

[6] P. Ribeiro, P. Milman and R. Mosseri, Phys. Rev. Lett. 93, 190503 (2004).

[7] A. Romanelli, R. Siri, V. Micenmacher. Phys. Rev. E 76, 037202 (2007). 
[8] A. Romanelli. Phys. Rev. E 78, 056209 (2008).

[9] A. Romanelli. Phys. Rev. A 80, 022102 (2009).

[10] H. Schomerus and E. Lutz, Phys. Rev. Lett. 98, 260401 (2007).

[11] H. Schomerus and E. Lutz, Phys. Rev. A 77, 062113 (2008).

[12] F.L. Moore, J.C. Robinson, C. Bharucha, P.E. Williams, M.G. Raizen, Phys. Rev. Lett. 73, 2974 (1994); J.C. Robinson, C. Bharucha, F. L. Moore, R. Jahnke, G. A. Georgakis, Q. Niu, M.G. Raizen, and B. Sundaram, ibid. 74, 3963 (1995); F.L. Moore, J.C. Robinson,C.F. Bharucha, B. Sundaram, M.G. Raizen, ibid. 75, 4598 (1995); J.C. Robinson, C. F. Bharucha, K. W. Madison, F. L. Moore, B. Sundaram, S. R. Wilkinson, and M. G. Raizen, ibid. 76, 3304 (1996).

[13] C.F. Bharucha, J.C. Robinson, F.L. Moore, Bala Sundaram, Qian Niu, and M.G. Raizen, Phys. Rev. E 60, 3881 (1999).

[14] W.H. Oskay, D.A. Steck, V. Milner, B.G. Klappauf, M.G. Raizen, Opt. Commun. 179, 137, (2000).

[15] J.F. Kanem, S. Maneshi, M. Partlow, M. Spanner and A. M. Steinberg, Phys. Rev. Lett. 98, 083004 (2007).

[16] M.C. Bañuls, C. Navarrete, A. Pérez, E. Roldán, and J.C. Soriano, Phys. Rev. A 73, 062304 (2006).

[17] A. Romanelli, Phys. Rev. A 80, 042332 (2009).

[18] F. M. Izrailev and D. L. Shepelyanskii, Sov. Phys. Dokl. 24, 996 (1979); Theor. Math. Phys. 43, 553 (1980).

[19] H. Ammann, R. Gray, I. Shvarchuck, and N. Christensen, Phys. Rev. Lett. 80, 4111 (1998).

[20] A. Romanelli, A.C. Sicardi Schifino, R. Siri, G. Abal, A. Auyuanet, and R. Donangelo. Physica A, 338, 395 (2004).

[21] A. Romanelli, A. Auyuanet, R. Siri, G. Abal, and R. Donangelo. Physica A, 352, 409 (2005). 